\title{
The need for review of CCIR curves for tropical Africa for effective television broadcasting planning
}

\author{
Zacchaeus Adesakin Adetona* \\ Electrical Engineering Department, \\ Federal Polytechnic, \\ P.M.B. 50, Ilaro, Ogun State, Nigeria, West Africa \\ E-mail: zachadetona@gmail.com \\ *Corresponding author
}

\section{Titus Olugbenga Koledoye}

Electrical Engineering Department, Yaba College of Technology,

P.M.B. 2011, Lagos State, Nigeria, West Africa

E-mail: koledoyetitus@gmail.com

\begin{abstract}
This paper examines how effective television broadcasting can be achieved in tropical African countries by developing new set of values for the International Radio Consultative Committee (CCIR) curve for use in sub-tropical Africa. Seven television stations were chosen in Lagos State Nigeria and the signal strength of each station was determined using a field strength metre in several locations around the state. The value obtained at a particular distance (in kilometres) is compared with values for free space and CCIR curve respectively. Computer software was then used to produce a graphical comparison between the CCIR curve and actual measurement. The results show that the CCIR curve is inadequate to guide effective television broadcasting in Nigeria and by extension, subtropical Africa. This result is considered valid for many tropical countries.
\end{abstract}

Keywords: television broadcasting; antenna; free-space; frequency re-use; coverage area; CCIR curve; electric field strength; co-channel interference; Africa.

Reference to this paper should be made as follows: Adetona, Z.A. and Koledoye, T.O. (2011) 'The need for review of CCIR curves for tropical Africa for effective television broadcasting planning', Int. J. Satellite Communications Policy and Management, Vol. 1, No. 1, pp.31-43.

Biographical notes: Zacchaeus Adesakin Adetona is a graduate of Electronic and Electrical Engineering from Obafemi Awolowo University, Ile-Ife, Osun State, Nigeria. He is presently studying to obtain MEng degree in Computer and Control Engineering at the University of Agriculture, Abeokuta, Ogun, State Nigeria and works as an Assistant Lecturer at the Federal Polytechnic, Ilaro, Nigeria.

Titus Olugbenga Koledoye is a graduate of Electrical Electronic Engineering from the University of Ilorin, Kwara State, Nigeria. He received his MSc in Telecommunications Engineering from the University of Lagos, Nigeria. He is presently working as a Lecturer at the Department of Electrical/Electronic Engineering, Yaba College of Technology, Lagos Nigeria. 


\section{Introduction}

Television (TV) broadcast as an aspect of communication engineering involves the transmission of both sound and pictures. Broadcasting generally, is a one-way communication, in that information is only transferred without a need for a reply. Figure 1 shows the block diagram of the basic TV broadcast system. It can be observed from the figure that TV broadcasting begins in a studio and the signal generated is coupled to a transmitter that radiates same into the atmosphere via an antenna. The radiated energy is picked up by a receiving antenna that is connected to a TV receiver that returns it into original audio and video sent from the TV station. For the received signal to be meaningful, a lot of factors come into play. One of such factors is the transmitting frequency.

For TV broadcasting, the range of frequencies from $41 \mathrm{MHz}$ to $960 \mathrm{MHz}$ which makes up the very high frequency (VHF) and the ultra-high frequency (UHF) respectively are used (WRTH, 1999; Grob and Herndon, 1999).

How the frequencies are allocated are as shown in Table 1. It is shown on the table that the TV broadcasting covers the above range of frequencies. The table also reveals the different TV bands in use.

The international planning of frequency allocation is achieved mainly through Administrative Radio Conference (ARC) of the International Telecommunication Union (ITU) in organisation empowered to carry out such assignments as:

1 allocation of frequency bands (i.e., specified services for frequency bands)

2 assignment of frequencies (for countries and international organisations)

3 regulation of the use of radio spectrum (in terms of standard rules of operation) coverage area restriction.

The ITU is made up of all member nations, on a worldwide or regional basis. The technical information required by the $\mathrm{ARC}$ is obtained from studies carried out by the International Radio Consultative Committee (CCIR). A similar study led to the putting together of this academic paper.

Figure 1 Block diagram of a TV broadcast system

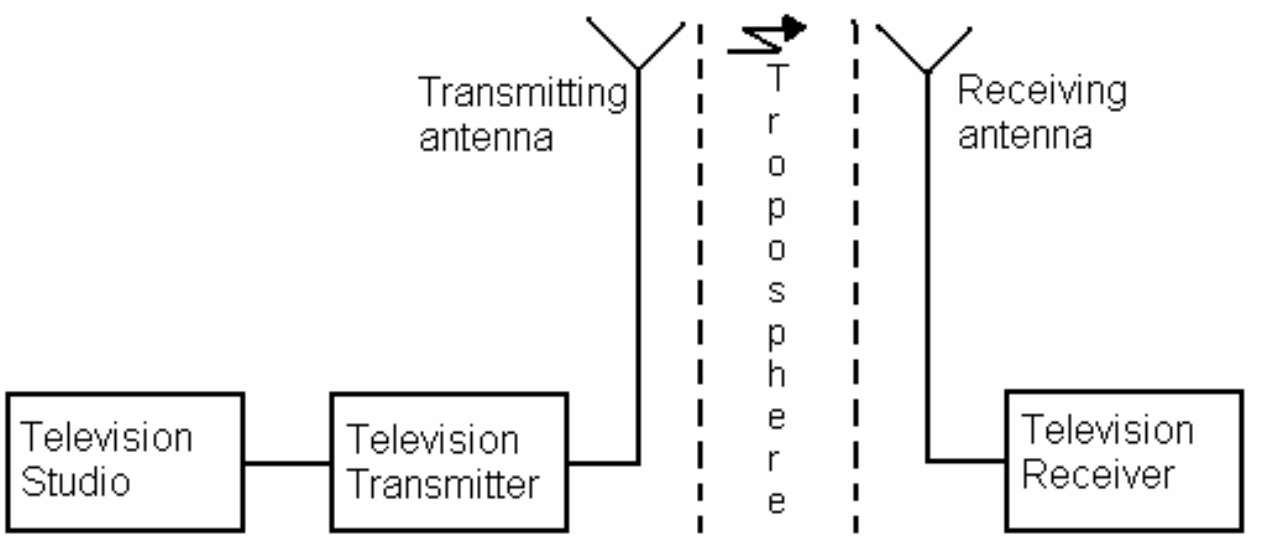


Table 1 Frequency allocation for TV broadcasting

\begin{tabular}{lccc}
\hline Frequency Spectrum & Band & Range $(\mathrm{MHz})$ & Service \\
\hline VHF & I & $41-68$ & TV BAND I \\
VHF & II & $85.5-100$ & FM SOUND \\
UHF & III & $175-215$ & TV BAND III \\
UHF & IV & $470-582$ & TV BAND IV \\
UHF & V & $582-960$ & TV BAND V \\
\hline \multicolumn{2}{r}{ Source: } & WRTH (1999)
\end{tabular}

The CCIR curve for TV broadcasting in sub-tropics is as old as the Geneva Conference on VHF/UHF Broadcasting in Africa, in 1963. According to the CCIR reports 239-5 and $370-4$ of 1982, the CCIR curves for temperate climate have been reviewed. Thus, it is clear that a review of the CCIR curves for sub-tropical Africa is essential and not out of place.

\subsection{Background}

The work of producing general curves for the purpose of determining TV broadcast transmission reach started as far back as the 1930s in many parts of the world. One of the earliest recognised was based on the so-called 'Cairo Curves' (NTIA, 1980). The curves were adopted by the CCIR in the year 1938. In the year 1946, the US Federal Communications Commission (FCC), in connection with their Clear Channel Hearing, Docket \#6741, also presented new sets of curves for propagation in North America (NTIA, 1980).

This work has continued until recent years in many parts of the world. Such measurements, however, were not extensively carried out in sub-tropical Africa continent especially in recent years. It is necessary to carry out such studies in the continent given the peculiarities in climatic conditions, vegetation and the terrain.

According to the NTIA (1980) report, "the final Acts of the Regional Administrative Low Frequency/Medium Frequency (LF/MF) Broadcasting Conference adopted the CCIR, Geneva 1974, sky-wave field strength prediction method for Australia and New Zealand in 1975". Further in 1979, Wang's method of prediction which was a modification of the CCIR Kyoto 1978 method was adopted for the USA.

\subsection{Consequences of reliance on inaccurate transmission curves}

When inaccurate transmission curves are employed to predict field strength for a transmitting station (whether TV or other forms of electronic communication), there is a very high possibility of occurrence of co-channel interference. Such was the case with the then NTA 2 Channel 5 TV station in Lagos State, Nigeria and Benin TV located in Cotonou, a city in the Republic of Benin, which shares boundary with Nigeria in West Africa.

Signals from the NTA 2 adversely interfered with that of Benin TV to the extent that viewers in parts of the Republic of Benin upon tuning in to Benin TV, oftentimes watched NTA 2 rather than Benin TV. This problematic issue was eventually resolved by reducing the transmitter power of the NTA 2 TV station. 
Similarly, on several occasions in parts of Lagos and Ogun States in Nigeria, a few TV stations transmitting from the Republic of Benin interfere with signals from some TV stations located in many areas in those two states. Further, in Nigeria signals from TV stations located in Lagos state can be received clearly in other states that are not within the same transmission zone as Lagos. This is not expected to be so if the field strength predictions were accurate. The foregoing informed the commencement of a study into the effectiveness of the CCIR curve predictions in the determination of transmission zones and setting up of a TV station.

\section{The need for radio spectrum allocation and re-use}

Within the radio spectrum is the VHF/UHF band which is internationally used for TV broadcast. The rate of exploration into radio spectrum in the recent century is alarming, as development in radio frequency propagation has grown with advances in technology. Thus, the available radio spectrum has been exploited more and more for all kinds of services both terrestrial and space, and the demand for frequency allocated is increasing steadily with no sign that it will cease to rise.

This TV spectrum cannot therefore go round all the TV stations of the world today if each station is to occupy a different channel. Also, as new TV stations come up in different parts of the world, there is a continuous increase in demand for frequency channels in the TV spectrum.

To meet up with the continuous increase in demand for frequency in the spectrum, the ITU put a radio spectrum regulation in place that ensures that, before a TV channel can be used in a country, approval must be given by the ITU. This is because the radio spectrum is a fixed commodity. In order to meet the needs of the generality of consumers and to overcome the problem of over-demanding, the ITU has resorted to repeating frequency allocations. This process is referred to as frequency re-use. This method ensures that demand is satisfied and, at the same time, ensures that TV transmission is free of co-channel interference through adequate regulations.

Since TV spectrum rationing equally affects sub-tropical Africa as it affects other parts of the world, it is imperative to encourage frequency allocation planning and management necessary to accommodate the demand for more channels. Such planning would benefit from the result of the study presented in this paper as it borders on real measurement necessary for good planning in sub-tropical Africa.

\subsection{TV broadcast coverage areas}

By the CCIR specifications, there are three categories of TV coverage area. There are:

- primary coverage area

- secondary coverage area

- fringe coverage.

The primary coverage area defines the area from the transmitting antenna to where the TV signals strength, E reduces to $2.5 \mathrm{mV}$. Secondary coverage area refers to the area where the signal strength of a transmitting antenna reduces from $2.5 \mathrm{mV}$ to $1 \mathrm{mV}$. Fringe 
coverage, according to the CCIR, refers to any area beyond the secondary coverage area where the TV signal can be received.

The three categories can be illustrated as shown in Figure 2 that follows:

Figure 2 Television coverage areas
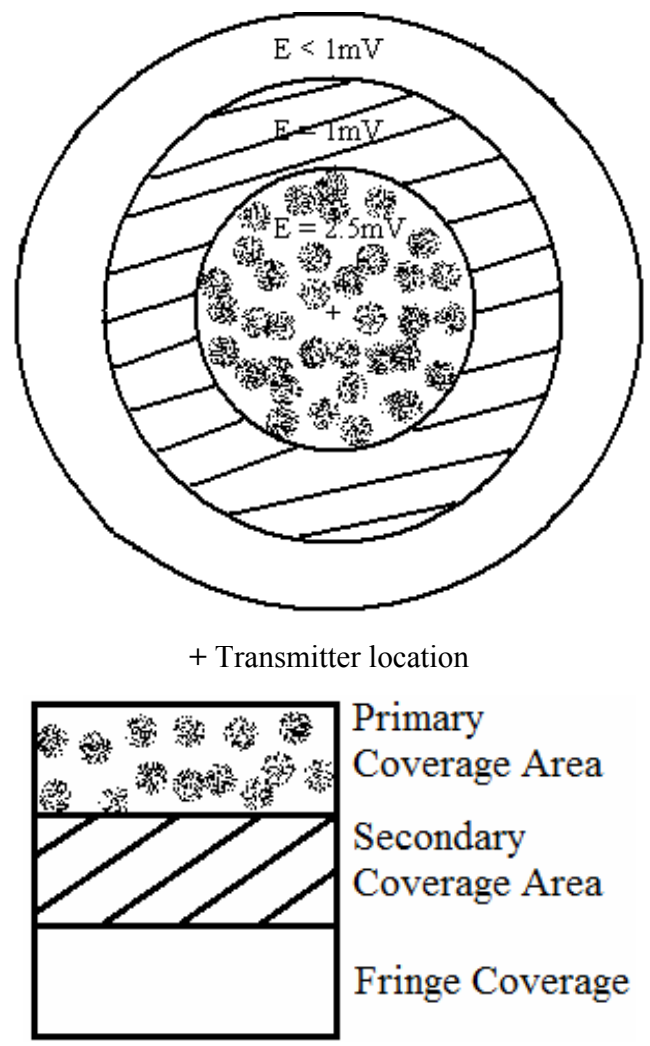

\section{Methods of determining TV coverage area}

Three approaches are available for determining the coverage area of a TV station. These are:

1 free space method

2 CCIR standard curve method

3 Field measurement

The three methods were employed and discussed as follow.

\subsection{Free space method}

This method assumes ideal situation, that is, interference and attenuation-free conditions. The coverage distance transmission loss, by space calculations, is defined as the ratio of 
the radio frequency power radiated in free space from an ideal loss-free isotropic transmitting antenna ( $G$, antenna gain equals 1 ) to the power available from an ideal, loss-free isotropic receiving antenna. This can be expressed mathematically (Hall, 1979) as:

$$
L_{b f}=\frac{P_{t}}{P_{r}}
$$

where $L_{b f}=$ basic free space transmission loss

$P_{t}=$ Effective radiated power

$P_{r}=$ Power available at the receiving antenna.

$$
P_{t}=4 \pi d^{2} P_{d}
$$

$d=$ coverage distance

$P_{d}=$ power flux per unit area.

$$
\begin{aligned}
P_{r} & =P_{d} A_{e} \\
A_{e} & =\frac{G \lambda^{2}}{4 \pi} \\
& =\frac{\lambda^{2}}{4 \pi}(G, \text { antenna gain equals } 1)
\end{aligned}
$$

$\lambda=$ radio frequency wavelength.

Therefore,

$$
P_{r}=\frac{P_{d} \lambda^{2}}{4 \pi}
$$

From equations (1), (2) and (5), we can deduce that:

$$
\frac{P_{t}}{P_{r}}=(4 \pi d / \lambda)^{2},(\text { Hall, 1979) }
$$

which is the same as

$$
\frac{V_{t}^{2} / \tau_{0}}{V_{r}^{2} / \tau_{0}}=(4 \pi d / \lambda)^{2}
$$

i.e.,

$$
\frac{V_{t}}{V_{r}}=\frac{4 \pi d}{\lambda}
$$

From the equation for transmission in free space (Green, 1979) given as:

$$
E=\left(\frac{30 P_{t}}{d}\right)^{1 / 2}
$$


where $E=$ electric field strength.

We have that

$$
E \times d=\left(30 P_{t}\right)^{1 / 2} \text { since } E \times d=V_{t}
$$

$V_{t}=$ transmitted signal voltage

$V_{r}=$ received signal voltage.

Using the signal voltage measured as reference from equation (6),

$$
d=\frac{V_{t}}{V_{r}}
$$

where

$\lambda=\frac{c}{f}$

$C=3 \times 10^{8} \mathrm{~m} / \mathrm{s}$

$f=$ transmission frequency.

Thus,

$$
d=\frac{V_{t}}{V_{r}} \cdot \frac{C}{4 \pi f}
$$

Therefore, the coverage area is the region within the coverage radius $d$ using the transmitting antenna as a reference point on the map.

Equation (8) was employed to determine the free space approach for each of the TV stations and the values obtained are given in Table 3. For example, for TV station $1, d$ is determined using the following given from Table 2 that $P_{t}=100 \mathrm{~kW}$ for the station:

$$
\begin{aligned}
V_{t} & =\sqrt{ }\left(30 P_{t}\right) \\
& =\sqrt{ }(30 \times 100) \quad \text { (Hall, 1979) } \\
& =1,732 \mathrm{~V}
\end{aligned}
$$

Now,

$$
\begin{aligned}
& C=3.0 \times 10^{8} \text { and } f=179.75 \times 10^{6} \text { from Table 2, using equation (8) we have: } \\
& \begin{aligned}
\frac{V_{t} \times C}{4 \pi f} & =\frac{1,732 \times 3 \times 10^{8}}{4 \times 3.142 \times 179.75 \times 10^{6}} \\
& =230 \mathrm{~V}
\end{aligned}
\end{aligned}
$$

Thus,

$$
d=\frac{230}{V_{r}}
$$


To determine the coverage area for the station at any location, the received signal voltage is used in equation (9). Similar method was employed for the free space and values for the other TV stations at various locations were obtained.

\subsection{CCIR standard curve method}

A number of factors do affect the transmission of electromagnetic waves from one point to another. Therefore, the transmission in the free space as determined by equation (8) will be affected. Such factors include the topography of the terrain of the coverage area, meteorological factors influence and the height of both transmitting and receiving antennas (Porges, 1988). Considering these factors, the CCIR produced propagation curves needed to aid the guide in the planning of broadcasting services. The CCIR curves determine the coverage distance relative to $\mathrm{dB}$ and it is based on effective radiated power (ERP) of $1 \mathrm{~kW}$.

$$
E I R P=P_{T} G_{T}
$$

where $P_{T}=$ transmitter output power

and $G_{T}=$ transmitting antenna gain.

Using the CCIR standard curves for both UHF and VHF propagation, the coverage distance was calculated for each location where the electric field strength measurement was taken. For an antenna height not available on the CCIR chart, method of linear interpolation given as follows was employed.

$$
\frac{h_{3}-h_{2}}{h_{2}-h_{1}}=\frac{X_{3}-X_{2}}{X_{2}-X_{1}}
$$

where

$h_{1}=$ height of antenna with value below the intended

$h_{2}=$ height of intended antenna

$h_{3}=$ height of antenna with value above $h_{2}$

$X_{1}=$ coverage distance of $h_{1}$ at specified $\mathrm{E}(\mathrm{dB})$

$X_{2}=$ the unknown coverage distance at the specified $\mathrm{E}(\mathrm{dB})$

$X_{3}=$ coverage distance of $h_{3}$ at specified $\mathrm{E}(\mathrm{dB})$ rel. to $1 \mu V$.

After $X_{2}$ is determined, the coverage distance d (ERP greater than $1 \mathrm{~kW}$ ) is determined by the following equation:

$$
d=\operatorname{ERP}(\mathrm{kW}) \times X_{2}
$$


Table 2 Transmission data for the seven TV stations

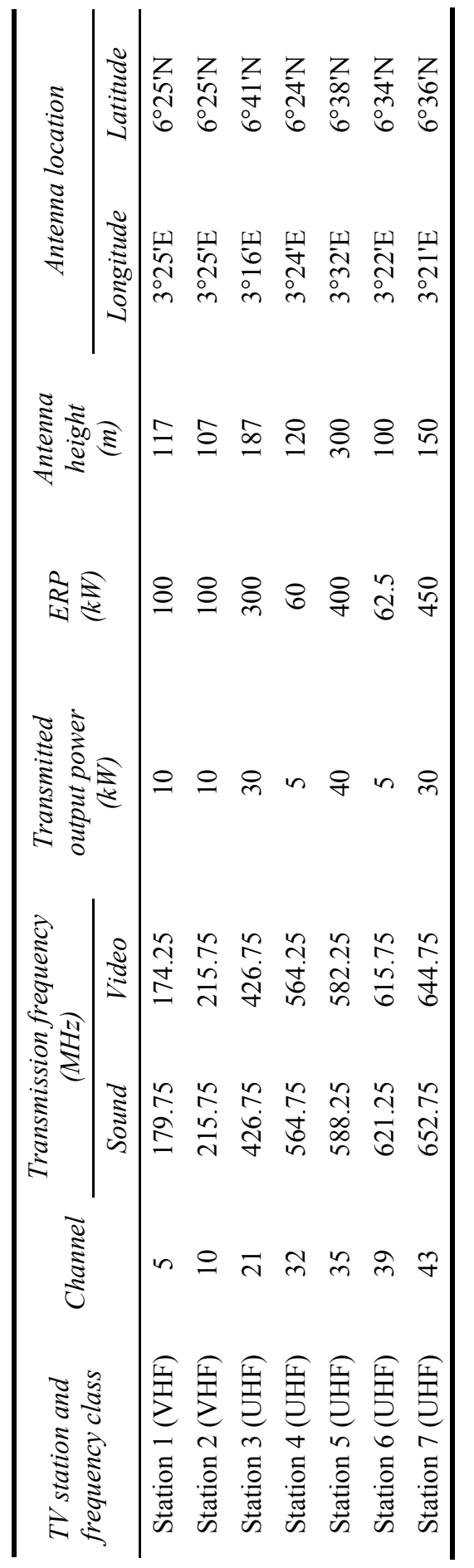


Table 3 Specified coverage distance for stations 1, 2 and 3

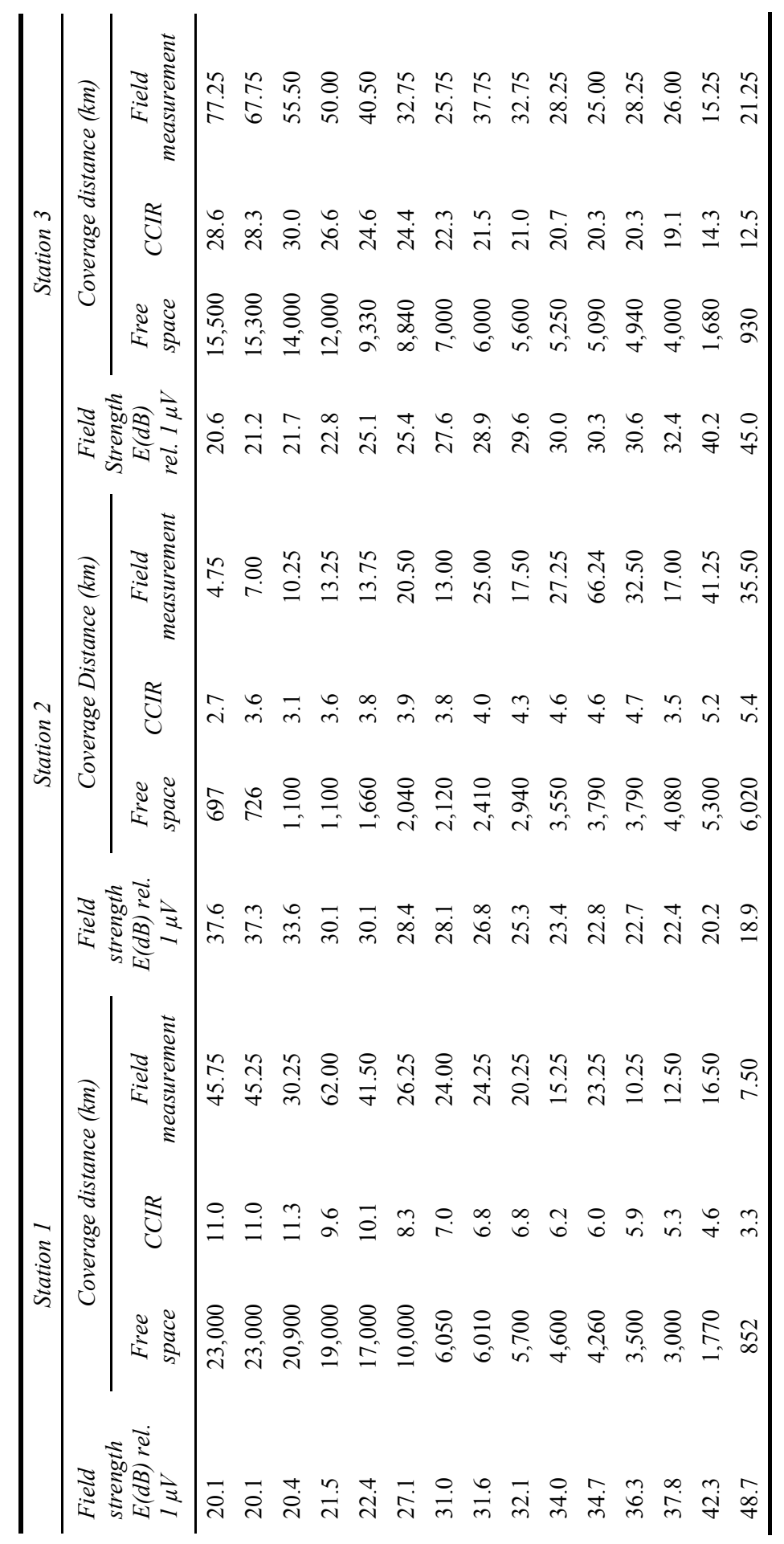




\subsubsection{Coverage distance calculations}

\section{Station 1}

antenna height, $h_{2}=117 \mathrm{~m}$

$\mathrm{ERP}=100 \mathrm{kw}$

from CCIR curve $h_{3}=150 \mathrm{~m}, h_{1}=75 \mathrm{~m}$

At Yaba location, $\mathrm{E}=37.8 \mathrm{~dB}$

$X_{3}=60 \mathrm{~m}, X_{1}=44 \mathrm{~m}$

Using equation (1)

$$
\frac{h_{3}-h_{2}}{h_{2}-h_{1}}=\frac{X_{3}-X_{2}}{X_{2}-X_{1}}
$$

i.e., $\frac{150-117}{117-75}=\frac{60-X_{2}}{X_{2}-44}$

from which $X_{2}=53 \mathrm{~m}$

$$
\begin{aligned}
\therefore d & =\operatorname{ERP} \times X_{2} \\
& =100 \times 53 \\
& =5.3 \mathrm{~km}
\end{aligned}
$$

2 Station 2

Antenna height, $h_{2}=183 \mathrm{~m}$

$\mathrm{ERP}=300 \mathrm{kw} h_{1}=150 \mathrm{~m}, h_{2}=300 \mathrm{~m}$

$\mathrm{E}=52.3 \mathrm{~dB}$ (Dopemu location)

$X_{3}=40 \mathrm{~m}, X_{1}=29 \mathrm{~m}$

Using equation (10)

$$
\begin{aligned}
X_{2} & =32.8 \mathrm{~m} \\
d & =\operatorname{ERP} \times X_{2} \\
& =300 \times 32.8 \\
& =9.8 \mathrm{~km}
\end{aligned}
$$

This was repeated for each station at all locations.

\subsection{Field measurement method}

In this approach, a practical method is employed to measure the electric strength at different locations in the coverage area of a TV station. The field strength metre was 
used to determine the transmitted signal strength at 50 different locations. The locations were then mapped out and joined together by a curve to trace out the coverage area.

How far the signal of a transmitting station travels depends, among many other factors, on the power of the transmitter (Porges, 1988). The higher the transmitter power, the farther the signal travels. Another factor that also affects the distance of travel is the height of the transmitting antenna above the sea level. These factors and various other necessary data of each of the seven TV stations examined in this study are provided in Table 2 .

\subsection{Results and analysis}

The CCIR curves are expected to be correct for $50 \%$ of locations for which they are employed (WRTH, 1999). The result of the CCIR method compared with the field measurement approach reveals that the CCIR curves proved inaccurate for all the locations. As can be seen in Tables 3, the values for coverage distance for the three stations chosen are wide apart for 15 selected locations. The free space value is not considered since the locations considered were within an urban area. It can be observed from the table that station 3 has longer transmission coverage due to its higher transmitter power of $300 \mathrm{~kW}$ as given in Table 2 .

The results recorded in Table 3 were used to generate radial curves using the Microsoft Excel software. The software produced a graph that shows a wide difference between the distance covered by the signal measured and the predicted coverage distance for the same values of signal strength. The difference is about $50 \mathrm{~km}$ in some instances as shown on Table 3 . Thus the graphs showed that the signal transmitted effectively covered the primary, secondary and the fringe broadcast areas well enough. One of the graphs is shown on Figure 3.

Figure 3 Broadcast coverage of station 1: CCIR compared with field measurement

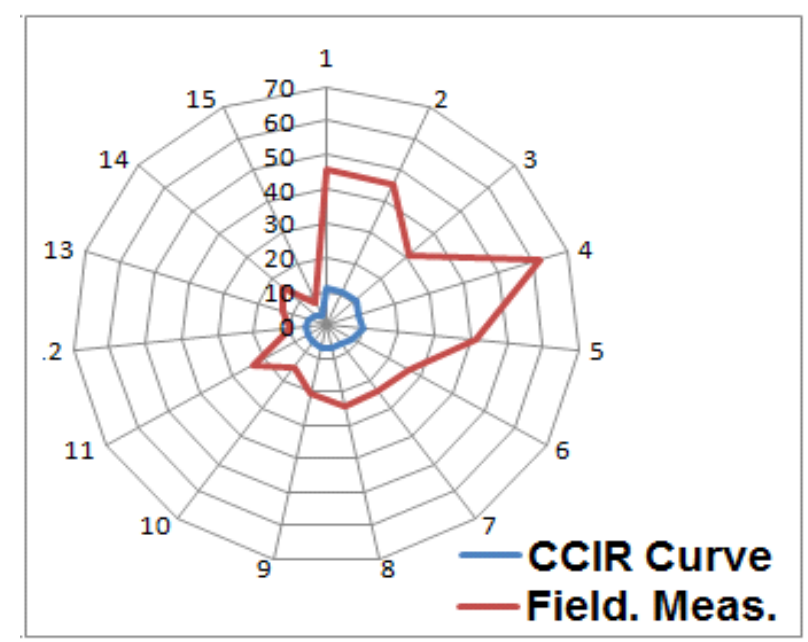




\section{Conclusions}

The differences observed in transmission coverage distance between the CCIR curve and field measurement approach is attributable to the age of the CCIR curves. The curves have been designed since 1953 and are expected to be valid for $50 \%$ of TV stations using them for broadcast planning. By this study, it is found that the curves do not provide such reliability any longer. The CCIR curves have not been modified since they were drawn while those of other continents have been modified (WRTH, 1999).

Thus there is the need to modify the CCIR curves for effective modern TV broadcast planning. The review of the CCIR curves will assist local and regional regulatory bodies to properly utilise available allocated frequency spectrum to a country or region. It is clear that the problem of co-channel interference was not expected with the present CCIR curves for sub-tropical Africa, but in cities or neighbouring states in a country where there are many TV stations springing up, causing a great demand on the frequency spectrum, allocating frequency becomes a difficult task to handle.

In this study, graphical method was used to demonstrate the inaccuracies of the CCIR curves. The final CCIR curves have not been drawn but are being proposed. It is suggested that further studies be carried out in different parts of the African continent that will result in a much more elaborate data collation. Such studies should also be carried out at different times of the year, that is, under different weather conditions and seasons.

With the availability of such an elaborate data, mathematical/statistical methods or a computer-based program (MATLAB is a good candidate) may be employed to generate the much needed curves for TV signals prediction.

\section{Acknowledgements}

The authors of this paper wish to express their gratitude to the following individuals and organisations for their contribution to the successful completion of the research work and compilation of the paper and its typing: Mrs Kenny Babalola of Lagos City Polytechnic, Ikeja, the Management of NTA 2 Channel 10 Lagos and AIT TV stations and Nigerian Broadcasting Commission (NBC) Lagos.

\section{References}

Green, D.G. (1990) Radio Systems Technology, John Wiley.

Grob, B. and Herndon, C.E. (1999) Basic Television and Video Systems, 6th ed., MacGraw-Hill.

Hall, M.P.M (1979) 'The effect of troposphere on radio communication', Institution of Engineer.

NTIA (1980) 'Comparison of available methods of predicting medium frequency sky-wave field strengths', Compiled by Margo PoKempner for The U.S. Department of Commerce.

Porges, F. (1988) The Design of Electrical Services, 3rd ed., Spon Press.

Wang, J.C.H. (1979) 'Medium-frequency skywave propagation in region 2', IEEE Trans. Broadcasting BC-25, pp.79-85.

WRTH (1999) World Radio TV Handbook, David G. Bobbett (Ed.), 53rd ed., Watson-Guptill Publications. 